\title{
Ecossistemas de conhecimento sobre Indústria 4.0 no Brasil: uma análise bibliométrica
}

\author{
Knowledge ecosystems on Industry 4.0 in Brazil: a bibliometric analysis \\ Mateus Rebouças Nascimento ${ }^{1}$ \\ ${ }^{1}$ Universidade Federal de Santa Catarina (UFSC), Florianópolis, SC, Brasil. ORCID: https://orcid.org/0000-0001-9211-327X
}

Autor para correspondência/Mail to: Mateus Rebouças Nascimento, maateusreboucas@gmail.com

Recebido/Submitted: 15 de junho de 2021; Aceito/Approved: 13 de setembro de 2021

Copyright (c) 2021 Nascimento. Todo o conteúdo da Revista (incluindo-se instruções, política editorial e modelos) está sob uma licença Creative Commons Atribuição 4.0 Internacional. Ao serem publicados por esta Revista, os artigos são de livre uso em ambientes educacionais, de pesquisa e não comerciais, com atribuição de autoria obrigatória. Mais informações em http://revistas.ufpr.br/atoz/about/submissions\#copyrightNotice.

\begin{abstract}
Resumo
Introdução: A produção de conhecimento na ciência se concretiza na interação colaborativa entre pesquisadores que investigam temáticas em comum, formando ecossistemas de conhecimento por meio de suas produções, neste sentido, o artigo objetiva mapear os principais atores (pesquisadores, universidades e agências de fomento) que constituem o ecossistema e os conhecimentos que o permeiam, em especial os centrados na indústria 4.0 em âmbito brasileiro. Método: Constitui uma pesquisa quantitativa, de cunho descritivo, utilizando técnicas bibliométricas mediante a uma análise dos trabalhos publicados sobre Indústria 4.0 na base de dados Web of Science nos últimos cinco anos, refinando a busca por país e selecionando o Brasil. Ao todo, 369 documentos produzidos no país foram analisados por meio do software métrico VOSviewer para construir redes de colaboração. Resultados: Destacam-se os autores Eduardo de Freitas Rocha Loures e Fernando Deschamps como principais produtores de conhecimento no ecossistema, bem como a CAPES e o CNPQ como atores norteadores no financiamento de pesquisas sobre a Indústria 4.0, as quais foram desenvolvidas no espaço da Universidade de São Paulo e Universidade Federal de Santa Catarina. Conclusão: Os ecossistemas de conhecimento na Indústria 4.0 são formados por universidades e agências de fomento públicas, que utilizam recursos para desenvolver no país tecnologias de inteligência artificial, internet das coisas, sistemas ciberfísicos, dentre outros conhecimentos norteadores.
\end{abstract}

Palavras-chave: Indústria 4.0; Análise bibliométrica; Ecossistemas de Conhecimento

\begin{abstract}
Introduction: The production of knowledge in science is materialized in the collaborative interaction between researchers who investigate common themes, forming knowledge ecosystems through their productions, in this sense, the article aims to map the main actors (researchers, universities and funding agencies) that constitute the ecosystem and the knowledge that permeates it, especially those centered on industry 4.0 in the Brazilian context. Method: It is a quantitative, descriptive research, using bibliometric techniques through an analysis of published works on Industry 4.0 in the Web of Science database in the last five years, refining the search by country and selecting Brazil. In all, 369 documents produced in the country were analyzed using the metric software VOSviewer to build collaboration networks. Results: The authors Eduardo de Freitas Rocha Loures and Fernando Deschamps stand out as the main producers of knowledge in the ecosystem, as well as CAPES and CNPQ as guiding actors in the financing of research on Industry 4.0, which were developed in the University space. of São Paulo and the Federal University of Santa Catarina. Conclusion: The knowledge ecosystems in Industry 4.0 are formed by universities and public development agencies, which use resources to develop in the country artificial intelligence technologies, internet of things, cyberphysical systems, among other guiding knowledge.
\end{abstract}

Keywords: Industry 4.0; Bibliometric analysis; Knowledge Ecosystems.

\section{INTRODUÇÃO}

A revolução no contexto da produção industrial 4.0, na atualidade, demanda a aplicação de mecanismos tecnológicos que contribuam para a produtividade em grande escala, digitalizando processos por meio de sistemas cibernéticos, inteligência artificial, internet das coisas, mecanismos estratégicos de big data, da inovação e do conhecimento que orientam as ações e decisões dos agentes na indústria 4.0, contribuindo para inserção da tecnologia nesses cenários.

Mediante processos desenvolvidos por diversos atores (agências de fomento, universidades, empresas, habitats de inovação, dentre outros), formam-se ecossistemas de conhecimento que propiciam a consolidação e o impacto produtivo dessa nova vertente tecnológica, por intermédio da relação entre atores na produção de conhecimento que perpassa a virtualização da alta tecnologia empregada, englobando a agregação de valor na execução.

Os ecossistemas de conhecimento consistem em organizações que compreendem diversos atores unidos por uma busca conjunta por conhecimento, de forma colaborativa para criação desse ativo (Järvi, Almpanopoulou, \& Ritala, 2018). Esses ambientes promovem interação e troca de conhecimento entre atores para melhorar a tomada de decisão em vastos cenários, podendo ser aplicado também aplicado também à indústria 4.0, no uso de redes para gerenciar processos.

O mapeamento de atores presentes em ecossistemas de conhecimento no contexto desta versão da indústria facilita a identificação do núcleo produtor de saberes sobre um determinado tema ou questão, interligando 
especialistas e agentes corporativos por meio de redes que "oferecem novas oportunidades para contribuir para a coevolução e o crescimento de outros ecossistemas, bem como de aglomerados geográficos e regionais ou regiões de aprendizagem" (Robertson, 2020, p. 16, tradução nossa).

O problema de pesquisa deste trabalho está pautado na necessidade de identificar os ecossistemas de conhecimento na indústria 4.0, mapear os principais atores que constituem esses ecossistemas e suas relações de criação de saber, além de reunir as principais referências sobre o assunto no Brasil, por meio de metodologias aplicadas nos estudos bibliométricos nos moldes propostos pelo campo da Ciência da Informação.

O objetivo geral desta pesquisa é analisar os atores que constituem o ecossistema de conhecimento sobre indústria 4.0 no Brasil, para isso, buscou-se: a) Mapear a produção científica sobre Indústria 4.0; b) Identificar os principais atores (pesquisadores, universidades e agências de fomento) e, c) Apresentar um mapa bibliométrico dos conhecimentos chave que são discutidos no debate científico sobre o assunto.

No plano científico, este estudo impacta o domínio da Ciência da Informação na medida em que contribui para uma análise metodológica da formação de ecossistemas de conhecimento, a qual pode ser posteriormente aplicada outros cenários além da indústria 4.0, possibilitando identificar reflexões inerentes a construção do conhecimento, bem como elementos externos que influenciam a consolidação desses conhecimentos no campo científico.

\section{REFERENCIAL TEÓRICO}

\section{Indústria 4.0: perspectivas para formação de ecossistemas de conhecimento}

Apresentada como a quarta revolução industrial, a indústria 4.0 consiste na digitalização abrangente e do uso da tecnologia na vinculação dos processos de produção, desde o pedido do cliente, passando pela criação de processos de produção, até a entrega dos serviços de produtos no mercado (Wilkesmann \& Wilkesmann, 2018).

M. O. Morais, Costa Neto, Santos, Cardoso, e Sacomano (2020, p. 5) acrescentam que este novo cenário vivenciado da indústria se manifesta na "existência de fontes inteligentes, uso extensivo de robótica, sistemas confiáveis de processamento e armazenamento de dados, além de monitoramento e controle das operações em tempo real". Compreende-se, assim que o controle da tecnologia empregada é fundamental para execução da produção em massa, de forma a monitorar os processos realizados.

Em termos práticos, a indústria 4.0 traz para si o que há de mais atual no que se refere à produção de bens, permeando a prospecção de inovação no cenário englobado, gerando novos mecanismos em produtos, esquematizando, assim, "um conjunto de tecnologias de ponta ligadas à internet com objetivo de tornar os sistemas de produção mais flexíveis e colaborativos" (Santos, Alberto, Lima, \& Charrua-Santos, 2018, p. 112)).

Lasi, Fettke, Kemper, Feld, e Hoffmann (2014) destacam conceitos fundamentais que definem a indústria 4.0, explicitando a importância da fabricação inteligente com o uso de sensores, atores e sistemas autônomos, também sistemas ciberfísicos na interação do nível físico e digital, auto-organização com foco na descentralização, sistemas de distribuição e aquisição conectados por vários canais diferentes, além da abordagem de inovação aberta, dentre outras adaptações fundamentais visando à eficiência na produção.

Sob essa perspectiva, Schwab (2017) apresenta quatro mudanças norteadoras que indústria tradicional sofre ao chegar em sua fase 4.0: alterações nas expectativas dos clientes, produtos mais inteligentes, novas formas de colaboração e a transformação do modelo operacional em digital por meio da tecnologia como catalizador de novas metodologias e processos

As tecnologias empregadas na indústria 4.0 integram percepções que contribuem para os processos produtivos, Vermulm (2018) destaca pontos principais em que elas colaboram para o desenvolvimento de produtos, apresentados na Tabela 1. 


\begin{tabular}{|l|l|}
\hline Tecnologia & Aplicação na Indústria 4.0 \\
\hline Big Data & $\begin{array}{l}\text { Processamento e armazenamento de grandes bases de dados estruturados e não } \\
\text { estruturados, fundamental para o tratamento computacional com o objetivo de analisar } \\
\text { processos e tomar decisões, mesmo que apenas uma parte dos dados seja analisada. }\end{array}$ \\
\hline $\begin{array}{l}\text { Computação em } \\
\text { Nuvem }\end{array}$ & $\begin{array}{l}\text { Utilizam computadores, servidores e toda infraestrutura necessária para processar e } \\
\text { armazenar bases de dados. Essa infraestrutura é compartilhada e acessada através da } \\
\text { internet, a partir de diversos dispositivos de acesso, tais como computadores, tablets e } \\
\text { celulares. }\end{array}$ \\
\hline $\begin{array}{l}\text { Inteligência } \\
\text { Artificial }\end{array}$ & $\begin{array}{l}\text { Permite que máquinas tomem decisões de forma autônoma, permite que produtos e } \\
\text { processos produtivos tomem decisões sem a interferência humana. }\end{array}$ \\
\hline $\begin{array}{l}\text { Internet das } \\
\text { Coisas }\end{array}$ & $\begin{array}{l}\text { Hardwares e softwares que viabilizam a interligação e a comunicação entre objetos, } \\
\text { podendo ser máquinas e equipamentos conectados entre si, ou bens de consumo } \\
\text { conectados com outros produtos. }\end{array}$ \\
\hline Robótica & $\begin{array}{l}\text { Equipamentos de automação industrial controlados automaticamente, podendo ser } \\
\text { programados e reprogramados à distância e com maior ou menor grau de autonomia, isto } \\
\text { é, sem a necessidade de intervenção humana. Permitem a tomada de decisão diante de } \\
\text { alterações das variáveis consideradas no processo de produção, e podem interagir com os } \\
\text { trabalhadores engajados em um determinado processo produtivo. }\end{array}$ \\
\hline $\begin{array}{l}\text { Sensores e } \\
\text { atuadores }\end{array}$ & $\begin{array}{l}\text { Identificam e registram informações sobre temperatura, pressão, umidade, presença de } \\
\text { produtos químicos, dimensões físicas, características físicas dos produtos etc. Essas } \\
\text { informações são processadas por controladores que acompanham e gerenciam a } \\
\text { produção. }\end{array}$ \\
\hline $\begin{array}{l}\text { Tecnologias de } \\
\text { Comunicação } \\
\text { sem Fio }\end{array}$ & $\begin{array}{l}\text { Sistemas constituídos por equipamentos, dispositivos, componentes e por softwares que } \\
\text { viabilizam a comunicação de voz e de dados sem fio, permitindo mobilidade dos agentes } \\
\text { dos processos produtivos. }\end{array}$ \\
\hline
\end{tabular}

Tabela 1. Tecnologias aplicadas no contexto da Indústria 4.0

Fonte: Adaptado de Vermulm (2018, p. 6).

Com essas tecnologias aplicadas à indústria 4.0, formam-se ecossistemas dentro dos quais um conjunto de atores que vivem em um espaço, interagem em torno de uma questão central como por exemplo, construir um aporte tecnológico para solução de um problema da sociedade, constituindo, assim, um sistema que contribui efetivamente no avanço de conhecimento, investigando questões de interesse que podem gerar novos mecanismos e funcionamentos industriais.

Assim, ao longo da revolução industrial 4.0, ocorre a interação colaborativa entre pesquisadores, empresas, universidades, agências de fomento e a sociedade como um todo, tendo como foco a inovação, além do conhecimento propício para formação de ecossistemas estruturados que geram novos saberes a partir de pesquisas científicas que buscam solucionar desafios da sociedade.

\section{Ecossistemas de conhecimento e os estudos métricos da informação}

Introduzido na Ecologia com objetivo de definir um conjunto de pessoas que constituem um ambiente de forma sistemática, o conceito de ecossistema perpassa diversos preceitos da sociedade de forma colaborativa, Amos Hawley destaca o termo aplicado para Ciências Sociais como "arranjo de dependências mútuas em uma comunidade pela qual o todo opera como uma unidade, no sentido de manter uma relação viável" (Hawley, 1986, p. 26).

No meio científico tecnológico, Valkokari (2015) mostra três modelos de ecossistemas que contribuem para identificação de atores no cenário empresarial, inovação e do conhecimento. A Tabela 2 traz suas respectivas linhas de base, relacionamentos, conectividade, atores, funções e a lógica que permeia o desenvolvimento de suas ações. 


\begin{tabular}{|l|l|l|l|}
\hline Vertentes & Ecossistemas de negócios & $\begin{array}{l}\text { Ecossistemas de } \\
\text { inovação }\end{array}$ & $\begin{array}{l}\text { Ecossistemas de } \\
\text { conhecimento }\end{array}$ \\
\hline $\begin{array}{l}\text { Base do } \\
\text { ecossistema }\end{array}$ & $\begin{array}{l}\text { Exploração de recursos } \\
\text { para agregar valor ao cliente }\end{array}$ & Cocriação de inovação & Exploração de conhecimento \\
\hline $\begin{array}{l}\text { Relacionamentos } \\
\text { e conectividade }\end{array}$ & $\begin{array}{l}\text { Relações de negócios } \\
\text { globais competitivas e } \\
\text { cooperativas }\end{array}$ & $\begin{array}{l}\text { Atores geograficamente } \\
\text { agrupados, diferentes } \\
\text { níveis de colaboração e } \\
\text { abertura }\end{array}$ & $\begin{array}{l}\text { Nódulos de conhecimento } \\
\text { descentralizados, sinergias } \\
\text { por meio da troca de } \\
\text { conhecimento }\end{array}$ \\
\hline Atores e funções & $\begin{array}{l}\text { Fornecedores, clientes e } \\
\text { empresas focais como um } \\
\text { núcleo, outros atores mais } \\
\text { vagamente envolvidos }\end{array}$ & $\begin{array}{l}\text { Formuladores de políticas } \\
\text { de inovação, } \\
\text { intermediários locais, } \\
\text { corretores de inovação e } \\
\text { organizações de } \\
\text { financiamento }\end{array}$ & $\begin{array}{l}\text { Institutos de pesquisa, } \\
\text { inovadores e } \\
\text { empreendedores } \\
\text { de tecnologia atuam } \\
\text { como nós de conhecimento }\end{array}$ \\
\hline Lógica de Ação & $\begin{array}{l}\text { Um ator principal que } \\
\text { opera como uma } \\
\text { plataforma que compartilha } \\
\text { recursos, ativos e } \\
\text { benefícios ou agrega outros } \\
\text { atores nas operações de } \\
\text { negócios em rede }\end{array}$ & $\begin{array}{l}\text { Atores geograficamente } \\
\text { próximos interagindo em } \\
\text { torno de centros } \\
\text { facilitados por atores } \\
\text { intermediários }\end{array}$ & $\begin{array}{l}\text { Um grande número de } \\
\text { atores que são agrupados } \\
\text { em torno da troca de } \\
\text { conhecimento ou de um } \\
\text { recurso central não } \\
\text { proprietário para o benefício } \\
\text { de todos os atores }\end{array}$ \\
\hline
\end{tabular}

Tabela 2. Características dos tipos de ecossistemas

Fonte: Adaptado de Valkokari (2015, tradução nossa).

Na visão apresentada por Valkokari (2015), os ecossistemas de conhecimento são prospectados por meio da exploração e uso de saberes organizado em núcleos com foco em trocar e compartilhar conhecimento por meio da colaboração entre universidades, empresas, agências de fomento e outros atores que formam redes de conhecimento, apoio, colaboração, dentre outras.

Miller (2015) aborda três elementos principais para consolidação de ecossistemas de conhecimento: recursos (elementos essenciais para produção de conhecimento e de informação), interações (relativas à informação para criar conhecimento) e aprendizagem (esfera informal de aprendizagem). O uso efetivo de recursos para integração da informação com foco na criação de conhecimento atinge uma esfera de aprendizagem, formada por pesquisadores, os quais estão vinculados às universidades e centros de pesquisa com suporte de agência de fomento para o desenvolvimento da ciência.

Para o mapeamento dos atores que constituem ecossistemas de conhecimento, os estudos métricos da informação podem ser fundamentais na identificação da construção, formação, interação e quantificação das pesquisas que envolvem ecossistemas, uma vez que são "métodos e técnicas de mensuração e avaliação quantitativa (estatístico-matemático) da produção, circulação e uso da informação" (Araújo, 2017, p. 44).

As autoras Grácio e Oliveira (2017, p. 105) reforçam as bases para o uso de métricas de informação:

[...] recursos quantitativos como método de análise, os estudos métricos da informação constituem o conjunto de conhecimentos relacionados à avaliação da informação produzida e são alicerçados na sociologia da ciência, na ciência da informação, matemática, estatística e computação. Referem-se aos estudos de natureza teórico-conceitual quando contribuem para o avanço do conhecimento da própria temática, propondo novos conceitos e indicadores, bem como reflexões e análises relativas à área.

Numa perspectiva genealógica, Curty e Delbianco (2020) destacam os marcos temporais e os principais autores que permeiam os estudos métricos e suas ramificações, mediante a uma análise da literatura desde o surgimento da bibliometria, o avanço para estudos cientométricos de disciplinas e aspectos econômicos da ciência, até a informetria com o uso da informação como elemento norteador de quantificação, além de outras métricas explicitadas na Figura 1. 


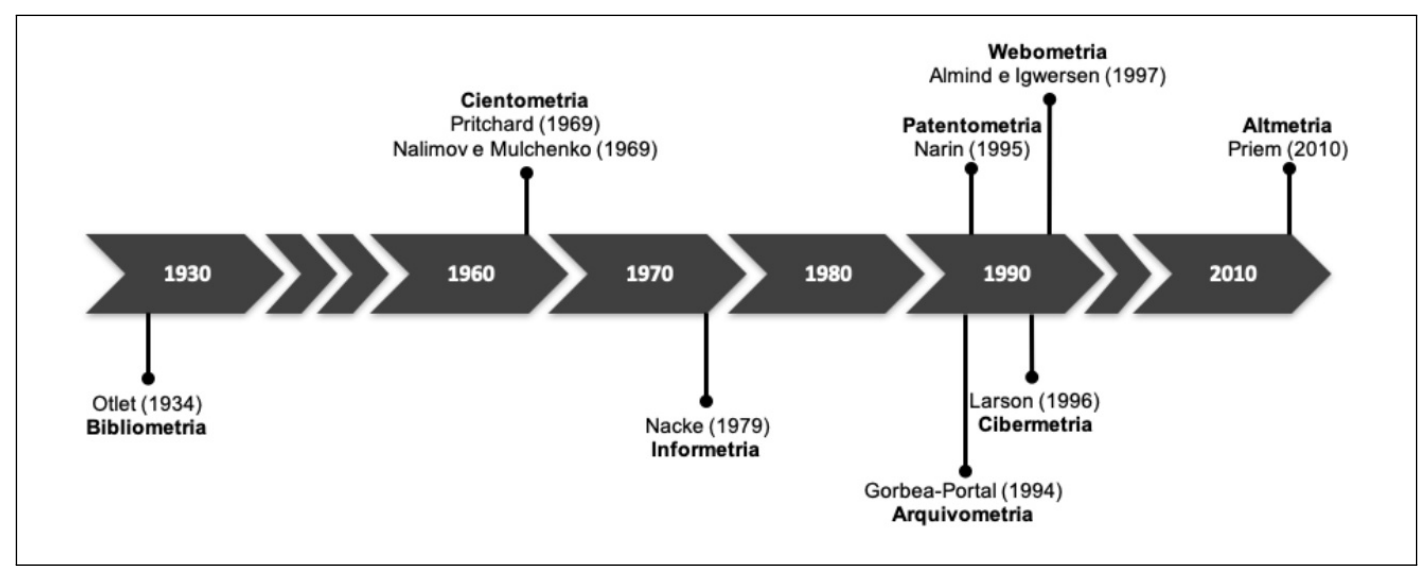

Figura 1. Genealogia dos Subcampos dos EMI Fonte:Curty e Delbianco (2020)

As ramificações dos estudos métricos da informação em subcampos consistem nas aplicações das métricas em diversas questões, como na produção de artigos (bibliometria), a consolidação da ciência (cientometria), informação em outros vertentes (informetria), uso de arquivos (arquivometria), registro de patentes (patentometria), o número de acesso na web (cibermetria e webometria), além das métricas alternativas que surgiram na última década (altmetria).

Por meio desses subcampos mencionados, é possível identificar agentes dos ecossistemas de conhecimento, destacando que a interação e construção na geração de novos conhecimentos faz com que os estudos métricos da informação "apresentem subsídios para quantificação e avaliação de diversas áreas do conhecimento, oportunizando metodologias sistemáticas para mensurar a produção desses ecossistemas de saberes." (Nascimento, 2021, p. 57).

Neste sentido, surge a necessidade de relacionar as ramificações dos estudos métricos da informação com o mapeamento de ecossistemas de conhecimento por meio das aplicações métricas, explicitando as interrelações que podem ser enxergadas em cada metria, contribuindo para formação e construção do conhecimento na ciência por meio da percepção dos conceitos teóricos e relações ecossistêmicas, conforme apresentado na Tabela 3. 


\begin{tabular}{|c|c|c|}
\hline Metria & Conceito & Aplicação em ecossistemas de conhecimento \\
\hline Bibliometria & $\begin{array}{l}\text { Registros impressos, citações, } \\
\text { agradecimentos, autores, usuários, } \\
\text { livros, revistas, artigos de revistas } \\
\text { (Vanti, 2005) }\end{array}$ & $\begin{array}{l}\text { Análise da produção de conhecimento que permeia } \\
\text { o ecossistema na geração de indicadores } \\
\text { bibliométricos para apresentar as temáticas } \\
\text { discutidas, os autores mais produtivos, periódicos } \\
\text { nos quais os conhecimentos estão sendo } \\
\text { publicizados, dentre outros. }\end{array}$ \\
\hline Cientometria & $\begin{array}{l}\text { Aspectos quantitativos da ciência } \\
\text { enquanto uma disciplina ou atividade } \\
\text { econômica, aplicada no } \\
\text { desenvolvimento de políticas científicas } \\
\text { (Macias-Chapula, 1998) }\end{array}$ & $\begin{array}{l}\text { Compreensão dos conhecimentos que perpassam o } \\
\text { ecossistema mediante a uma análise das disciplinas } \\
\text { de áreas do conhecimento e o financiamento } \\
\text { econômico prospectado para cada área. }\end{array}$ \\
\hline Informetria & $\begin{array}{l}\text { Todo tipo de informação em qualquer } \\
\text { tipo de suporte, comunicações formais } \\
\text { e informais, entre quaisquer grupos } \\
\text { sociais, de qualquer forma e em } \\
\text { qualquer canal (Araújo, 2015) }\end{array}$ & $\begin{array}{l}\text { Análise das comunicações informacionais na } \\
\text { interação entre pesquisador, universidade e } \\
\text { agência de fomento na mensuração da informação } \\
\text { que perpassa a geração de conhecimento no } \\
\text { ecossistema }\end{array}$ \\
\hline Patentometria & $\begin{array}{l}\text { Indicadores patentários com vistas a } \\
\text { identificar atividades de inovação e } \\
\text { tecnologias nos países, através das } \\
\text { informações tecnológicas contidas nos } \\
\text { documentos de patentes } \\
\text { (S. P. Morais \& Garcia, 2014) }\end{array}$ & $\begin{array}{l}\text { Registros dos conhecimentos-chaves nos } \\
\text { documentos de patentes para prospecção de } \\
\text { inovação no ecossistema e o mapeamento de quais } \\
\text { temáticas estão fomentando mais tecnologia }\end{array}$ \\
\hline Webometria & $\begin{array}{l}\text { Método de estudo quantitativo e } \\
\text { qualitativo das informações disponíveis } \\
\text { na web através dos links dos sites, } \\
\text { (Silva, 2016) }\end{array}$ & $\begin{array}{l}\text { Mapeamento dos conhecimentos discutidos no } \\
\text { ecossistema por meio dos links em sites, visando a } \\
\text { identificar os mais destacados na web, além da } \\
\text { relação com outros conhecimentos contidos em } \\
\text { outras páginas }\end{array}$ \\
\hline Altmetria & $\begin{array}{l}\text { Comunicação em microblogs, blogs, } \\
\text { comunidades virtuais, grupos de } \\
\text { discussão e sites de redes sociais } \\
\text { (Araújo, 2015) }\end{array}$ & $\begin{array}{l}\text { Identificação dos conhecimentos comunicados por } \\
\text { atores dos ecossistemas em comunidades virtuais } \\
\text { como redes sociais acadêmicas e grupos mediante } \\
\text { a aplicação de métricas alternativas }\end{array}$ \\
\hline
\end{tabular}

Tabela 3. Subcampos dos EMI e aplicação em ecossistemas de conhecimento Fonte: Os autores (2021).

Com isso, observa-se que, a cada ramificação dos estudos métricos da informação, é possível identificar atores e temáticas que constituem os ecossistemas de conhecimento, tendo em vista que são núcleos complexos formado por um sistema de pessoas, universidades, centros de pesquisa, organizações, tecnologias e processos no qual o conhecimento é construído, criado, interpretado e absorvido e aplicado em processos (Thomson, 2007).

\section{PROCEDIMENTOS METODOLÓGICOS}

Para compreensão da temática sobre indústria 4.0 e ecossistemas de conhecimento voltados à aplicação dos estudos métricos da informação, realizou-se, inicialmente, uma leitura dos fundamentos teóricos principais por meio de um levantamento da produção científica no Portal de Periódicos da Capes, para compor o referencial teórico da pesquisa, selecionando as principais fontes que sustentam o aporte teórico da investigação.

Após esse levantamento, e tendo como foco identificar e analisar os agentes dos ecossistemas de conhecimento sobre indústria 4.0, foi feita uma busca sistematizada na base de dados Web of Science (WoS) com o termo "industry 4.0", filtrando os trabalhos publicados no recorte temporal dos últimos cinco anos (2015-2020), considerando que os trabalhos publicados nesse intervalo abordam os conhecimentos emergentes e tecnologias mais recentes sobre indústria 4.0. O objetivo da busca nas bases de dados foi identificar pesquisadores, universidades e agências de fomento brasileiras e quais os conhecimentos-chave gerados por esses agentes.

A coleta foi realizada na primeira semana de junho de 2021, recuperando um total 8.020 documentos. A busca foi refinada por país (Brasil), uma vez que o objetivo é analisar a produção brasileira sobre a temática, depois da filtragem por Brasil totalizou-se 369 artigos publicados nos últimos cinco anos fruto de esforços de pesquisadores brasileiros, constituindo assim como elemento de análise estes trabalhos publicizados para traçar a extração dos atores que constituem os ecossistemas de conhecimento sobre indústria 4.0.

A WoS foi escolhida como base para extração do corpus desta pesquisa porque faz referência a vários bancos de dados interdisciplinares, com a aplicação de critérios de qualidade em avaliações de impacto, englobando também periódicos de acesso aberto (Clarivate Analytics , 2017), compreendendo que o tema indústria 4.0 pode 
ser aplicado em múltiplas áreas do conhecimento, como Artes e Humanidades, Ciências da Vida, Ciências Sociais, Tecnologia, entre outras.

A identificação dos dados quantitativos a respeito dos pesquisadores, universidades e agências de fomento que mais se destacam como atores no ecossistema sobre indústria 4.0 ocorreu por meio da análise dos resultados extraídos pela base de dados na janela "analisar resultados", propiciando o mapeamento e posterior correlação com as redes de conhecimento e colaboração prospectadas mediante a aplicação da bibliometria.

Para geração dos grafos de análise das temáticas principais que formam ecossistemas de conhecimento sobre indústria 4.0, utilizou-se o software métrico VOSviewer ${ }^{1}$ na versão 1.6.16, identificando os conhecimentos-chave no assunto por meio da coocorrência de palavras, unificando os termos duplicados para analisar de forma efetiva a rede de conhecimento que permeia o ecossistema, considerando o mínimo de duas ocorrências da palavra para compor o mapa.

O layout selecionado para apresentação dos grafos de conhecimento no software foi o de overlay visualization, sabendo que este modelo explicita por meio do recorte temporal e de cores a abrangência e destaque de determinada temática em respectivo ano, conforme a legenda apresentada no canto inferior direito do grafo, apontando a incidência da cor baseado na ocorrência anual.

O software métrico VOSviewer foi escolhido ${ }^{2}$, pois, de forma facilitada, propicia a criação de mapas e extração de informações em base de dados de uma rede como a WoS, construindo, então, "redes de publicações científicas, periódicos científicos, pesquisadores, organizações de pesquisa, países, palavras-chave ou termos" (Van Eck \& Waltman, 2018, p. 3).

\section{RESULTADOS}

Os resultados da pesquisa são pautados na apresentação do mapeamento dos principais atores que constituem o ecossistema de conhecimento sobre indústria 4.0 no Brasil: pesquisadores que mais se destacam, universidades da indústria 4.0, agências de fomento, além dos conhecimentos-chave voltados às tecnologias que norteiam o desenvolvimento da indústria no Brasil.

Quanto aos principais produtores de conhecimento sobre indústria 4.0 na Web of Science, expostos na Tabela 4, infere-se que a grande parte dos autores mais produtivos estão vinculados a universidades instaladas na região nordeste, sul e suedeste do Brasil, considerando os estados do Paraná, Santa Catarina e Rio Grande do Sul. Ao todo, foram contabilizados 45 trabalhos publicados por Loures, Deschamps, Frazzon e Frank.

\begin{tabular}{|l|l|l|l|l|}
\hline Autor & Trabalhos & Filiação & Índice H & Citações \\
\hline $\begin{array}{l}\text { Eduardo de F. R. } \\
\text { Loures }\end{array}$ & 14 & Pontifícia Universidade Católica do Paraná & 6 & 603 \\
\hline Fernando Deschamps & 11 & Pontifícia Universidade Católica do Paraná & 5 & 43 \\
\hline Enzo M. Frazzon & 11 & Universidade Federal de Santa Catarina & 11 & 493 \\
\hline Alejandro G. Frank & 9 & Universidade Federal do Rio Grande do Sul & 4 & 51 \\
\hline Fabricio Junqueira & 9 & Universidade de São Paulo & 6 & 188 \\
\hline Paulo E. Miyagi & 9 & Universidade de São Paulo & 11 & 429 \\
\hline Ivanovitch Silva & 8 & Universidade Federal do Rio Grande do Norte & 11 & 430 \\
\hline
\end{tabular}

Tabela 4. Principais produtores de conhecimento sobre indústria 4.0

Fonte: Os autores (2021).

Com objetivo de verificar o reconhecimento dos autores na WoS, mapeou-se o índice $h^{3}$ e o número de citações na base de dados em relação ao total de trabalhos publicizados pelos autores, inclusive indo além da temática da indústria 4.0, ressaltando que Frazzon, Miyagi e Silva possuem o maior índice dentre os mais produtivos, por conta de outras produções vinculadas a assuntos que que não se referem à indústria 4.0, mas sim estudos emergentes em outras áreas de conhecimento que esses pesquisadores investigam.

Em relação ao número de citações, constata-se como gerador de conhecimento central o pesquisador da Eduardo de F. R. Loures, vinculado à Pontifícia Universidade Católica do Paraná (PUC-PR), totalizando 603 citações, baseado em suas produções na ciência, seguido de Enzo M. Frazzon da Universidade Federal de Santa Catarina (UFSC) com 493 citações e o índice $h$ igual a 11, o mais elevado entre os autores mapeados. Entende-se, assim

\footnotetext{
${ }^{1} \mathrm{O}$ software VOSviewer pode ser instalado gratuitamente disponível para Microsoft Windows, MAC Os X e outros sistemas: https://www.vosviewer.com/download

${ }^{2}$ Para conhecer outros softwares métricos disponíveis no mercado para estudos voltados às metrias da informação: Moreira, Guimarães, e Tsunoda (2020). Qual ferramenta bibliométrica escolher? um estudo comparativo entre softwares. P2P e Inovação, 6(2), 140-158. doi: 10.1016/j.joi.2017.08.007.

${ }^{3} \mathrm{O}$ índice $h$ tem como objetivo quantificar a produtividade e o impacto da produção científica de pesquisadores por meio dos artigos mais citados.
} 
que os trabalhos do pesquisador têm visualização e impacto, assim como os de Miyagi e Silva, que possuem mesmo índice $h$.

Quanto às afinidades dos atores mapeados com o ecossistema de conhecimentos sobre indústria 4.0, destacam-se que as linhas de pesquisa com foco no desenvolvimento de técnicas para modelagem, análise e controle de sistemas baseados nos conceitos de indústria 4.0, considerando-se as inovações inerentes que dão suporte a essa fase da produção industrial à indústria 4.0. Exemplos de assuntos pesquisados são: internet das coisas, orientação à serviços, realidade virtual, dentre outras dimensões, além da construção de conceitos, métodos e técnicas para a gestão e a tomada de decisão em sistemas produtivos, entendendo que os preceitos desta quarta vertente percorrem uma manutenção inteligente no processo industrial.

Em termos do compromisso dos principais produtores de saber com as questões da indústria 4.0, nossa análise evidencia os esforço de Loures e Deschamps em colaborar com o campo por meio da criação de uma startup, a Go44 Avaliações e Soluções para Indústria 4.0, com objetivo de realizar avaliações de maturidade para implementação da indústria 4.0 em empresas, utilizando padrões de desenvolvimento tecnológico em nível mundial, demonstrando, assim, que os conhecimentos produzidos na academia se transferem para outros autores.

Os ecossistemas de conhecimento sobre indústria 4.0 são formados por estes atores pesquisadores identificados na tabela, os quais constroem saberes por meio de suas investigações científicas vinculadas a universidades, criando e gerando conhecimento para sociedade. A Figura 2 apresenta as instituições referência sobre indústria 4.0 no Brasil.

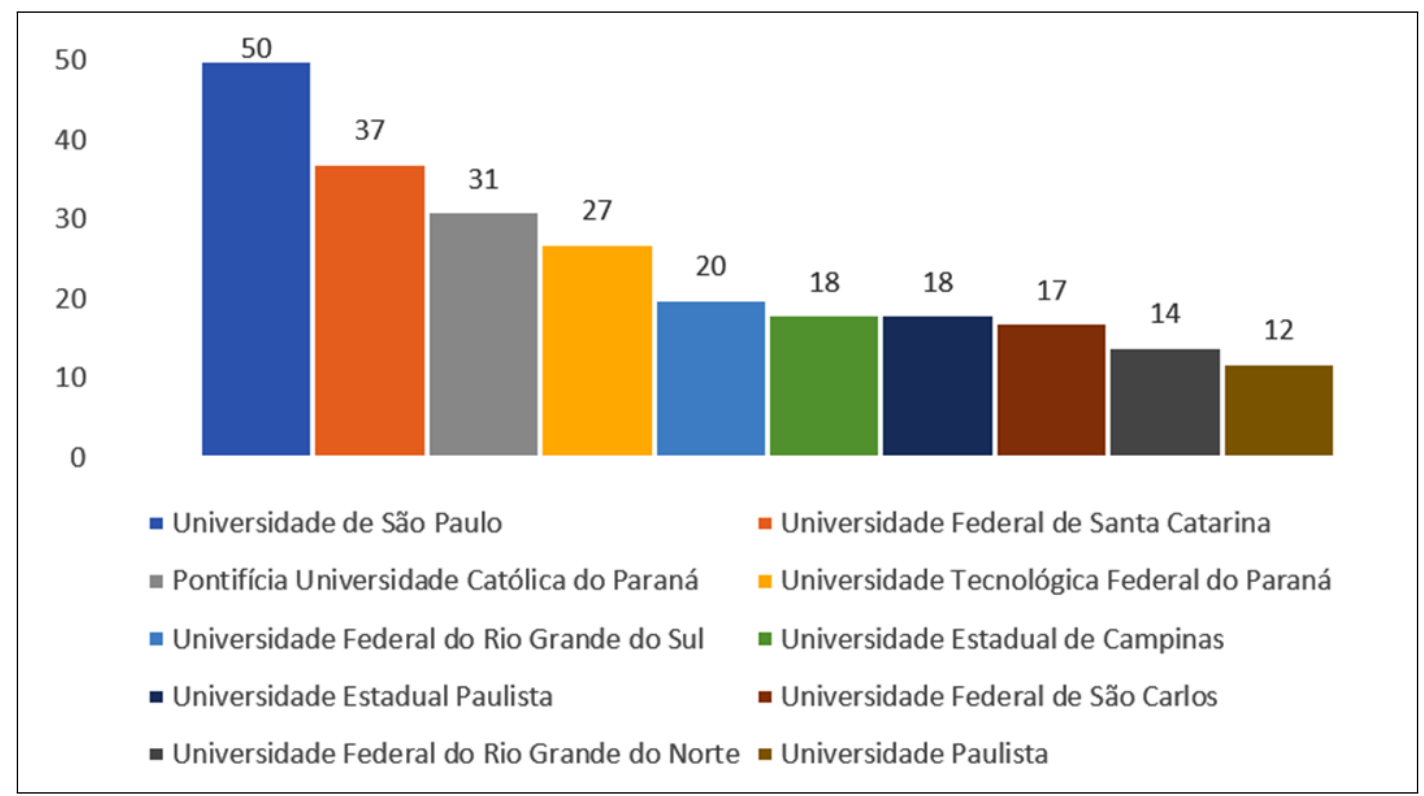

Figura 2. Universidades referência na vertente 4.0

Fonte:Dados da pesquisa (2021)

A instituição de ensino brasileira que mais publica trabalhos sobre a vertente 4.0 das indústrias é a Universidade de São Paulo (USP) totalizando 50 comunicações, neste ranqueamento, a PUCPR aparece em terceiro lugar com 37 publicações. Por um lado, a PUCPR tem os pesquisadores mais produtivos segundo a WoS; por outro, a USP tem um maior número de autores que publicizam conhecimentos no ecossistema.

No contexto das universidades brasileiras, o estado que possui mais instituições de referência em comunicações científicas sobre indústria 4.0 é São Paulo, totalizando cinco universidades no ranking de maiores produtoras de conhecimento, evidenciando a dominância paulista no total da produção brasileira de conhecimento, com colaboração científica internacional e nacional (Fapesp, 2010).

Já o fomento às pesquisas e tecnologias para indústria 4.0 ficam, em grande parte, a cargo de entidades públicas como a Coordenação de Aperfeiçoamento de Pessoal de Nível Superior (CAPES), o Conselho Nacional de Desenvolvimento Científico e Tecnológico $(\mathrm{CNPq})$, assim como a Fundação de Amparo à Pesquisa de São Paulo, consolidando como visto no gráfico anterior, as universidades paulistas como produtoras de conhecimento no ecossistema. 


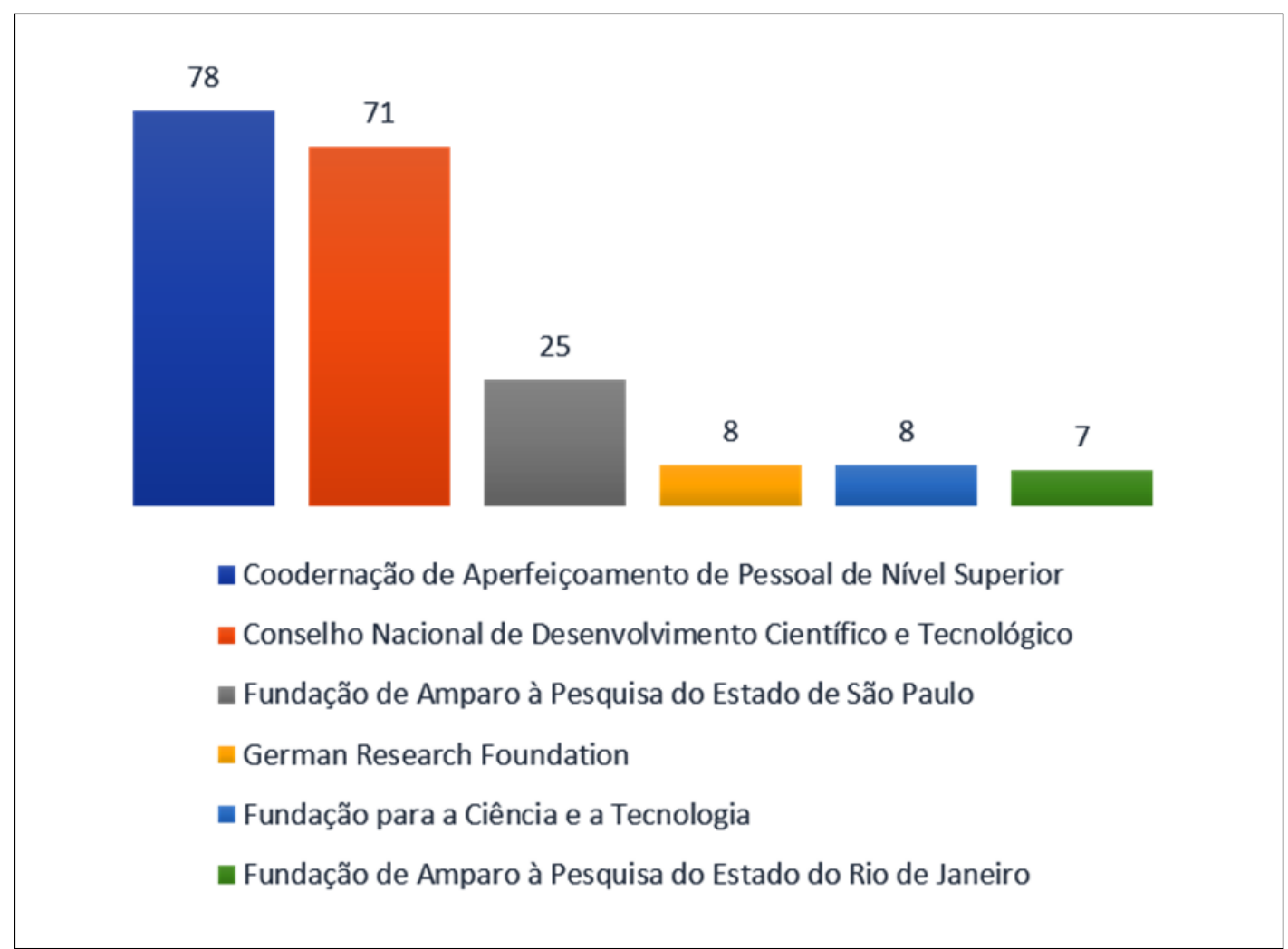

Figura 3. Agências de fomento financiadores da indústria 4.0 Fonte:Dados da pesquisa (2021)

Todavia, apesar a da prevalência de agências públicas brasileiras, existem também atores internacionais no ecossistema e que fomentam o conhecimento como a German Research Foundation da Alemanha e a Fundação para Ciência e Tecnologia de Portugal, mostrando que a indústria 4.0 possui visibilidade por parte de colaboradores externos ao Brasil, os quais incentivam estudos em tecnologias que podem ser utilizadas na geração e criação de conhecimento, agregando valor ao processo realizado nas indústrias.

Com a criação, geração e uso de tecnologia nos processos industriais dessa revolução 4.0, têm-se a necessidade de mapear quais são os conhecimentos-chave que constituem o ecossistema, as disciplinas inerentes, bem como os assuntos que se relacionam ao desenvolvimento de saberes e metodologias que melhoram a produtividade no ambiente industrial. Esses conceitos principais são apresentados na Figura 4, na forma de mapa gerado pelo software VOSviewer. 


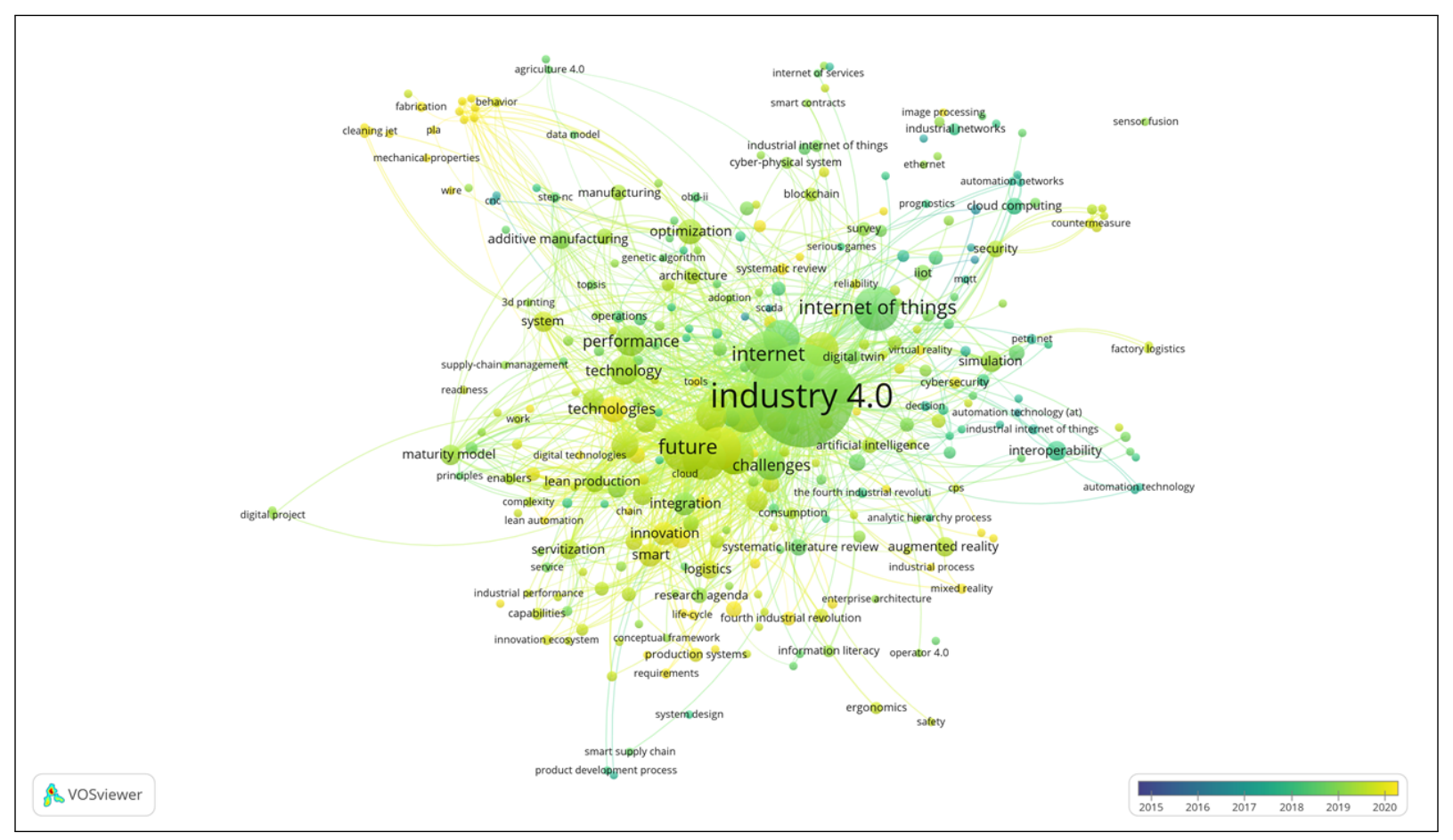

Figura 4. Mapa de conhecimentos chave para indústria 4.0 Fonte:Dados da pesquisa (2021)

A temática da indústria 4.0 está associada a diversos conhecimentos de tendências tecnológicas como internet, futuro, desempenho, inovação, lógica, sistemas ciberfísicos, gamificação, dentre outras tecnologias, corroborando as aplicações indicadas por Vermulm (2018), e enfatizando o uso da internet das coisas e inteligência artificial no planejamento da produção industrial atualizada. O sistema global da internet no mapa de conhecimento na visão 4.0 pode ser analisado conforme a interconexão digital no ambiente da internet das coisas. Entenda-se manufatura aditiva, otimização dos processos, serviços de análise de tecnologia, desenvolvimento de produtos inteligentes, segurança da informação, cidade inteligente, sistemas multiagentes, além de estudos envolvendo algoritmos para solucionar um determinado problema. Ao verificar a relação dos atores mapeados na Tabela 4 com os conhecimentos que são gerados no ecossistema sobre indústria 4.0, nota-se que esses saberes são voltados $\mathrm{a}:$

- avaliação diagnóstica para análise de capabilidade e maturidade do setor industrial frente aos requisitos da indústria 4.0;

- capacitações para criação de planos de diretor de tecnologias orientado para o aumento da maturidade nos conceitos da dimensão 4.0;

- desenvolvimento e aplicações de tecnologias emergentes focadas para o desenvolvimento da indústria de alimentos e bebidas, na área de embalagens cartonadas e tecnologia de envase;

- gestão de sistemas de produção no contexto 4.0.

Nos ecossistemas de conhecimento relevantes para indústria 4.0, o futuro próximo prevê a aplicação de tecnologias voltadas à arquitetura, produção e automação industrial compacta, projetos digitais, modelos de maturidade na gestão de processos, além da inovação - fundamental para construção de novos conhecimentos e práticas ecossistêmicas, de modo a garantir a qualidade máxima, com menor índice de paradas não programadas na linha de produção, por meio dos conhecimentos reforçados no mapa apresentado.

\section{CONCLUSÃO}

Ao fim deste artigo, pode-se entender que a indústria 4.0 está transformando a linha de produção com o uso de tecnologias que contribuem para aumentar a produtividade, oferecendo métodos e qualidade aos envolvidos no processo, sensibilizando os atores desse campo a adotarem novas práticas, formando ecossistemas estruturados colaborativamente com conhecimentos-chave para eficiência industrial.

Portanto, o mapeamento de atores em ecossistemas de conhecimento sobre a quarta revolução industrial contribui para identificar quem são as principais referências que podem contribuir para solução de diversos problemas na indústria. Por meio das metodologias de estudos métricos da informação, foi possível apontar os pesquisadores 
mais produtivos, bem como as universidades e agências de fomento mais proeminentes no Brasil e no exterior, e como esses agentes criam e melhoram conhecimentos e práticas da indústria 4.0 no país.

De forma resumida, podemos colocar que os ecossistemas de conhecimento sobre indústria 4.0 no Brasil são compostos por pesquisadores vinculados às principais universidades brasileiras, uma vez que estes desenvolvem pesquisas em múltiplas áreas do conhecimento, enquanto buscam financiamento para esses trabalhos.

Em relação aos conhecimentos-chave para a indústria 4.0 no Brasil, conclui-se que estão focados na aplicação da tecnologia na produção de forma automatizada, com logísticas que proporcionam o uso da inteligência artificial por meio de sistemas ciberfísicos, integrando a inovação na busca por melhorias para operações na indústria, visando ao melhor uso de recursos, evitando desperdícios e utilizando a tecnologia a favor da otimização de ações no ambiente industrial. 


\section{REFERÊNCIAS}

Araújo, R. F. (2017). Estudos métricos da informação na web e o papel dos profissionais da informação. Biblioteca Universitária, 2, 42-64. Recuperado de https://periodicos .ufmg.br/index.php/revistarbu/article/view/3074

Clarivate Analytics . (2017). Web of science. Recuperado de http://wilearncap.asuscomm.com:81/wikipedia en all _novid_2017-08/A/Web_of_Science.html

Curty, R. G., \& Delbianco, N. R. (2020). As diferentes metrias dos estudos métricos da informação: Encontros Bibli: revista eletrônica de biblioteconomia e ciência da informação, 25, 01-21. doi: 10.5007/1518-2924.2020.e74593

Fapesp. (2010). Análise da produção científica a partir de publicações em periódicos especializados. In Indicadores de ciência, tecnologia e inovação em são paulo 2010. São Paulo: Fapesp. Recuperado de https://fapesp.br/indicadores/2010/ volume1/cap4.pdf

Grácio, M. C. C., \& Oliveira, E. F. T. (2017). A pesquisa brasileira em estudos métricos da informação: proximidade entre pesquisadores de destaque e áreas afins. Informação ES Sociedade, 27(2), 105-116. Recuperado de https:// periodicos.ufpb.br/ojs/index.php/ies/article/view/32483

Hawley, A. H. (1986). Human ecology: A theoretical essay. Chicago: University of Chicago Press.

Järvi, K., Almpanopoulou, A., \& Ritala, P. (2018). Organization of knowledge ecosystems: Prefigurative and partial forms. Research Policy, 47(8), 1523-1537. doi: 10.1016/j.respol.2018.05.007

Lasi, H., Fettke, P., Kemper, H.-G., Feld, T., \& Hoffmann, M. (2014). Industry 4.0. Business 8 Information Systems Engineering, 6(4), 239-242. doi: 10.1007/s12599-014-03344

Macias-Chapula, C. A. (1998). O papel da informetria e da cienciometria e sua perspectiva nacional e internacional. Ciência da Informação, 27(2), 132-140. doi: 10.1590/s010019651998000200005

Miller, F. Q. (2015). Experiencing information use for early career academics' learning: a knowledge ecosystem model. Journal of Documentation, 71 (6), 1228-1249. doi: 10.1108/jd-04-2014-0058

Morais, M. O., Costa Neto, P. L. O., Santos, O. S., Cardoso, A. P., \& Sacomano, J. B. (2020). A evolução da qualidade na indústria 4.0. Research, Society and Development, 9(10), 1-29. doi: 10.33448/rsd-v9i10.8634

Morais, S. P., \& Garcia, J. C. R. (2014). O estado da arte da patentometria em periódicos internacionais da ciência da informação. Encontro Brasileiro de Bibliometria e Cientometria, 4. Recuperado de http://hdl.handle.net/ 20.500.11959/brapci/45400

Moreira, P. S. C., Guimarães, A. J. R., \& Tsunoda, D. F. (2020). Qual ferramenta bibliométrica escolher? um estudo comparativo entre softwares. P2P e Inovação, 6(2), 140-158. doi: $10.1016 /$ j.joi.2017.08.007

Nascimento, M. R. (2021). Ecossistemas de conhecimento e a ciência da informação: os estudos métricos da informação na mensuração do conhecimento científico. In Sustentabilidade informacional em ecossistemas de conhecimentos. Manaus, AM: Editora da Universidade Federal do Amazonas. Recuperado de http://riu.ufam.edu.br/handle/prefix/5856

Robertson, J. (2020). Competition in knowledge ecosystems: A theory elaboration approach using a case study.
Sustainability, 12(18), 7372. doi: 10.3390/su12187372

Santos, B. P., Alberto, A., Lima, T. D. F. M., \& CharruaSantos, F. M. B. (2018). Indústria 4.0: desafios e oportunidades. Revista Produção e Desenvolvimento, 4 (1), 111-124. doi: 10.32358/rpd.2018.v4.316

Schwab, K. (2017). The fourth industrial revolution. Nova Iorque: Crow Business.

Silva, I. O. (2016). Webometria e a análise de redes sociais. Revista ACB: Biblioteconomia em Santa Catarina, 21(2), 294-308. Recuperado de https://revista.acbsc.org.br/racb/ article/view/1137

Thomson, A. J. (2007). How should we manage knowledge ecosystems? using adaptive knowledge management. In Sustainable forestry: From monitoring and modelling to knowledge management and policy science. Wallingford: CABI.

Valkokari, K. (2015). Business, innovation, and knowledge ecosystems: How they differ and how to survive and thrive within them. Technology innovation management review, 5(8), 17-24. Recuperado de https://timreview.ca/article/ 919

Van Eck, N. J., \& Waltman, L. (2018). Vosviewer manual. Holanda: Universiteit Leiden.

Vanti, N. A. P. (2005). Os links e os estudos webométricos. Ciência da Informação, 34(1), 78-88. doi: 10.1590/s010019652005000100009

Vermulm, R. (2018). Políticas para o desenvolvimento da indústria 4.0 no brasil. São Paulo: IEDI. Recuperado de http://web.bndes.gov.br/bib/jspui/handle/1408/15486

Wilkesmann, M., \& Wilkesmann, U. (2018). Industry 4.0 - organizing routines or innovations? VINE Journal of Information and Knowledge Management Systems, 48(2), 238-254. doi: 10.1108/vjikms-04-2017-0019

Como citar este artigo (APA):

Nascimento, M. R. (2021). Ecossistemas de conhecimento sobre Indústria 4.0 no Brasil: uma análise bibliométrica. AtoZ: novas práticas em informação e conhecimento, 10(3), 1 - 12. Recuperado de: http:// dx.doi.org/10.5380/atoz.v10i3.81513 